\title{
EVALUATION OF HORMONAL PATTERN AND INDEX OF OXIDATIVE STRESS IN NORMAL WEIGHT WOMEN WITH
}

POLYCYSTIC OVARY SYNDROME.

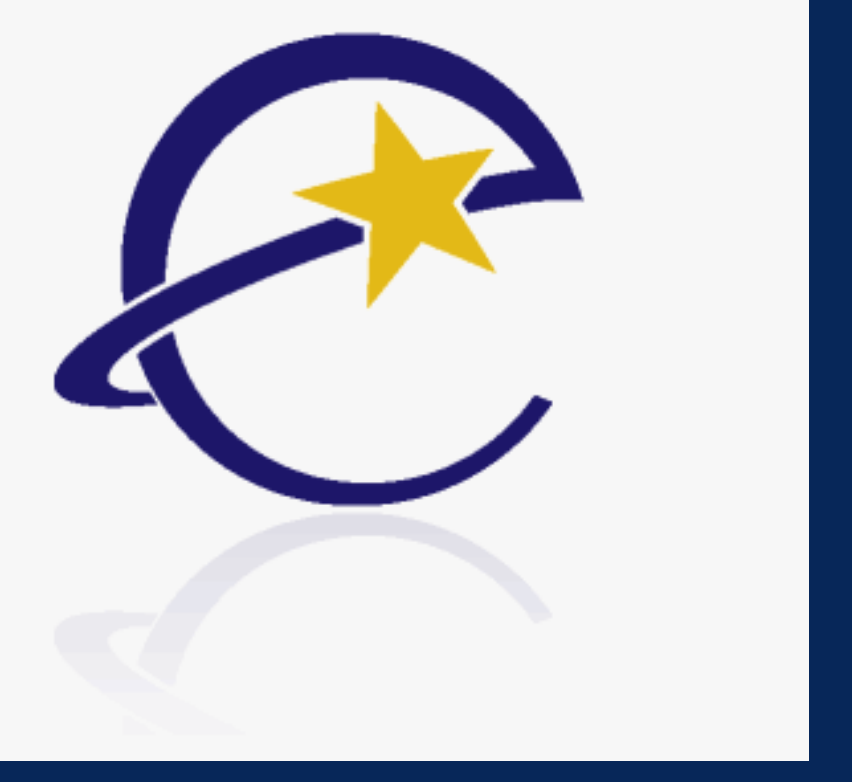

\section{Di Segni ${ }^{1}$, S. Raimondo ${ }^{1}$, Fato $^{3}$, C. Bergamini², V. Fuoco ${ }^{1}$, M. Cammarano D. Romuald ${ }^{4}$, A. Lanzone ${ }^{4}$, A. Pontecorvi ${ }^{1}$, A. Mancini ${ }^{1}$ \\ 1Dept. Of medical Sciences, Division of Endocrinology, Catholic University of Sacred Heart, Rome, Italy ${ }^{2}$ CIRI-HST , University of Bologna, Bologna, Italy \\ ${ }^{3}$ Dept. Of Pharmacology and Biotechnology (FaBiT), University of Bologna, Bologna, Italy ${ }_{4}^{4}$ Dept. Of Obstetrics and Gynecology, Catholic University of Sacred Heart, Rome, Italy}

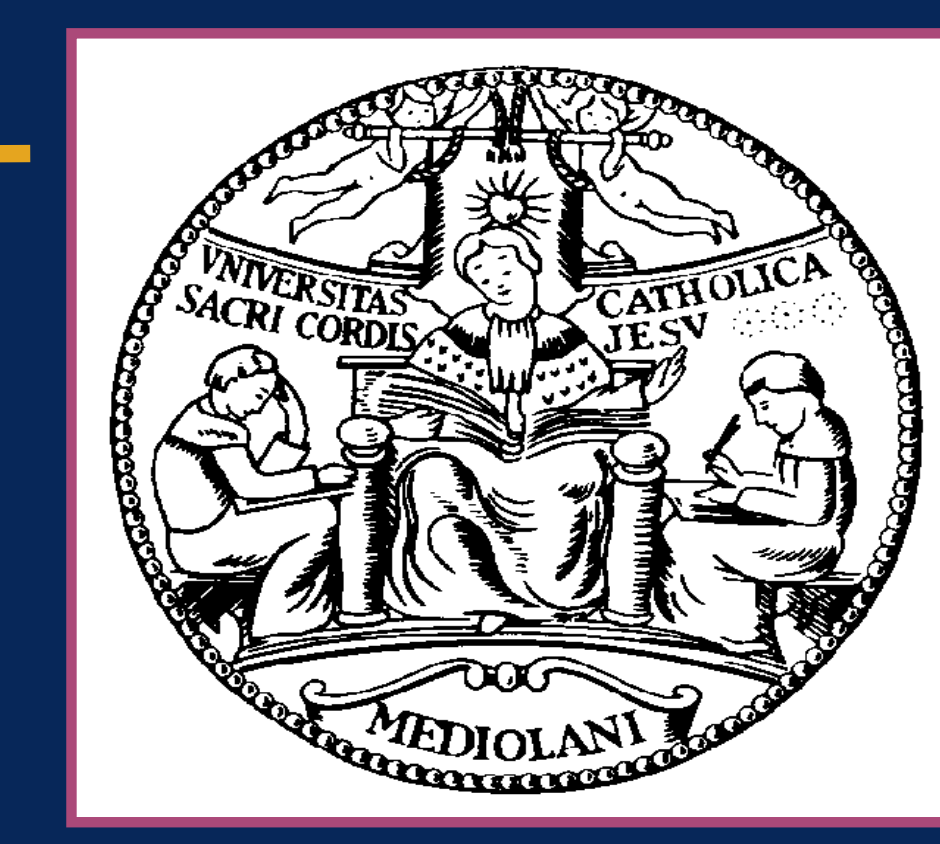

\section{Objective}

It is well known that insulin resistance (IR) is associated with polycystic ovary syndrome (PCOS). Oxidative stress (OS) is, in turn, related to $I R$, with a vicious cycle. PCOS patients presented higher circulating concentrations of oxidative stress products such as homocysteine, malondialdehyde, an increase of superoxide dismutase and reduction of antioxidants such as glutathione and paraoxonase-1 activity. Most studies however concerned obese PCOS subjects.

In order to investigate parameters of OS in normal weight PCOS and the relationships with hormonal and metabolic parameters, we have evaluated the concentrations of Coenzyme $Q_{10} \quad\left(C \circ Q_{10}\right)$, a component of mitochondrial respiratory chain, also endowed with antioxidant properties, in plasma of PCOS and normal menstruating women. Also malondialdheyde (MDA), a product of lipid peroxidation, was evaluated.

\section{Methods}

We have evaluated - $n=7$ PCOS patients, age 20-25 ys, mean BMI $24.8 \pm 2.6$ and

- $\mathrm{N}=7$ normal menstruating women, age 20-25 ys, mean BMI

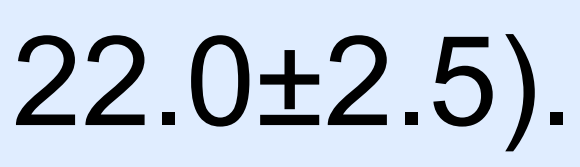

$\mathrm{CoQ}_{10}$ levels were determined by HPLC according to Takada et al. and MDA levels were determined spectrophotometrically at $535 \mathrm{~nm}$ by TBARS assay.

Hormonal studies included evaluation of: TSH, fT3, fT4, IGF-1, Testosterone, DHEAS, Androstenedione (by CLIA method) and HOMA index. (Table1)

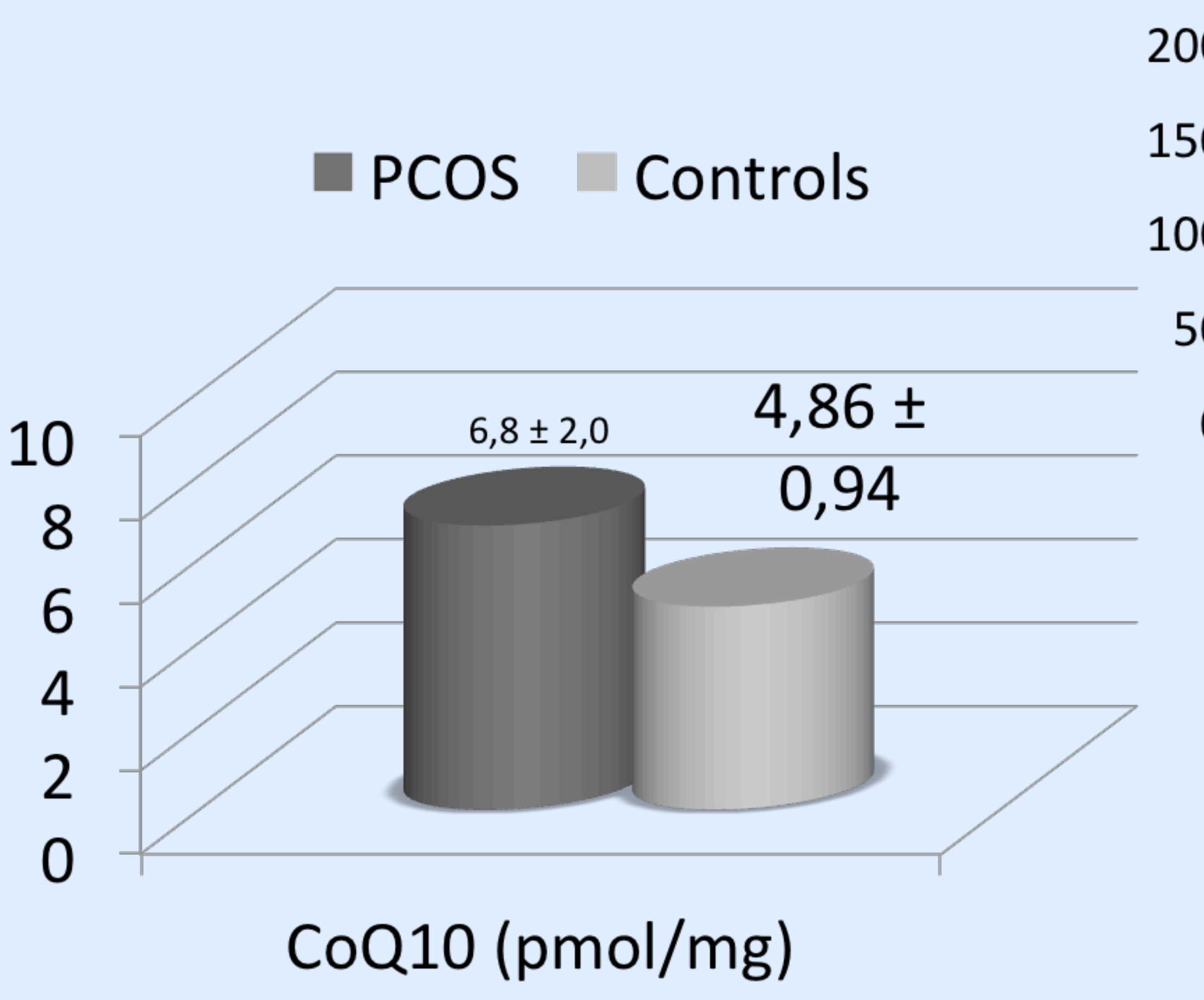

Results

We did not find a significant difference in MDA (in PCOS patiens mean $\pm E S: ~ 7020 \pm 2474 \mathrm{pmol} / \mathrm{ml}$ vs $12380 \pm 2198.9$ in controls) and $\mathrm{CoQ}_{10}(577.2 \pm 41.6 \mathrm{pmol} / \mathrm{ml}$ vs 495.6 638.8 ). (Fig.1a-b)

PCOS patients showed a trend toward a lower fT3 levels $(2.8 \pm 0.07$ vs $3.3 \pm 0.12 \mathrm{pg} / \mathrm{ml})$ and higher IGF- 1 levels $(303 \pm 9.3$ vs $279.2 \pm 46.1 \mathrm{ng} / \mathrm{ml}$ ).

PCOS Controls

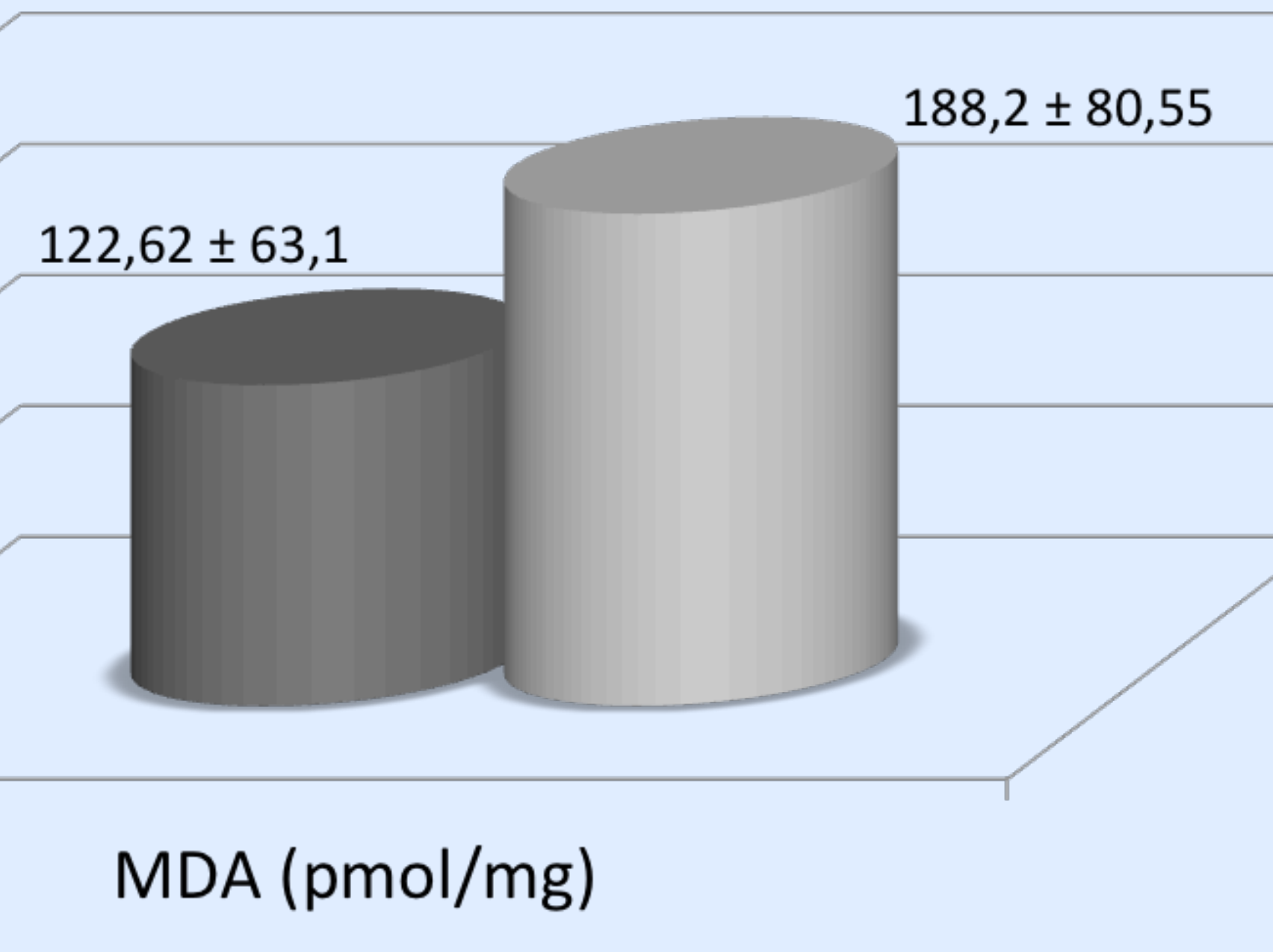

Fig. $1 a-b$

\section{Conclusions}

Table 1. MeantES in PCOS patients and controls

\begin{tabular}{|c|c|l|l|l|l|l|c|}
\hline & TSH $(\boldsymbol{\mu U I} / \mathbf{l})$ & $\begin{array}{l}\mathbf{f T 3}(\mathbf{p g} / \\
\mathbf{m l})\end{array}$ & $\mathbf{f T 4}(\mathbf{p g} / \mathbf{m l})$ & $\mathbf{I G F - 1}(\mathbf{n g} / \mathbf{m l})$ & $\mathbf{T}(\mathbf{n g} / \mathbf{m l})$ & DHEAS $(\mathbf{n g} / \mathbf{m l})$ & A $(\mathbf{n g} / \mathbf{m l})$ \\
\hline $\begin{array}{c}\text { PCOS } \\
(\mathbf{n}=7)\end{array}$ & $1.71 \pm 0.07$ & $2.8 \pm 0.07$ & $9.65 \pm 0.20$ & $303 \pm 9.68$ & $0.77 \pm 0.09$ & $3478 \pm 69.91$ & $2.11 \pm 0.23$ \\
\hline $\begin{array}{c}\text { Controls } \\
(\mathbf{n}=7)\end{array}$ & $279.25 \pm 0.14$ & $3.3 \pm 0.13$ & $11.12 \pm 0.29$ & $279.25 \pm 47.87$ & $0.55 \pm 0.03$ & $2742.33 \pm 265.75$ & $2.06 \pm 0.21$ \\
\hline
\end{tabular}

These preliminary data suggest that $O S$ is not simply related to $I R$ in normal weight PCOS but there is a complex interplay between hormones influencing follicular growth. They need to be extended to furnish further insight into the mechanisms of hyperandrogenism in such a condition and to give a rationale for a therapeutic employment of antioxidants in PCOS. 